\title{
Impact of self phase modulation on the performance of Brillouin distributed fibre sensors
}

\author{
S. M. Foaleng ${ }^{* a}$, F. Rodríguez ${ }^{\mathrm{b}}, \mathrm{S}$. Martin Lopez ${ }^{\mathrm{b}}$, M. González Herráez ${ }^{\mathrm{c}}$, L. Thévenaz ${ }^{\mathrm{a}}$ \\ ${ }^{a}$ Ecole polytechnique Fédérale de Lausanne (EPFL), Institute of Electrical Engineering, \\ STI-GR-SCI-LT Station 11, CH-1015, Lausanne, Switzerland; \\ ${ }^{\mathrm{b}}$ Instituto de Física Aplicada, CSIC. C/ Serrano 144, Madrid 28006, Spain \\ ${ }^{c}$ Departamento de Electrónica, Universidad de Alcalá, Campus Universitario s/n, Spain
}

\begin{abstract}
The spectral broadening of the pump pulse through self phase modulation in a time domain distributed Brillouin sensor is demonstrated to have a non-negligible detrimental effect, leading to a doubling of the effective gain linewidth after some $20 \mathrm{~km}$ in standard conditions. The theoretical modeling is fully confirmed by experimental results.
\end{abstract}

Keywords: Brillouin sensors, fibre optics sensors, non linear effects, self phase modulation.

\section{INTRODUCTION}

For at least two decades Brillouin fibre sensors have attracted a great interest in the fibre sensing community for their temperature and strain monitoring capability ${ }^{1,2,3}$ supporting a pluridisciplinary field of application (civil engineering, oil \& gas industry, security border parameter and intrusion detection). While improving the spatial resolution of these sensors has attracted most of the research effort ${ }^{4}$, the detection range is also a key parameter that must be as long as possible. In time-domain distributed Brillouin sensors pulses are used to interrogate the local interaction in the fibre. The accuracy on the measurand is scaled by the spectral spreading of the effective gain, which at its turn is given by the convolution between the pulse spectrum and the natural Brillouin gain spectrum (BGS). According to standard time-bandwidth relations, the Gaussian pulse is presumably the best candidate for this interrogation when compared to other profiles (rectangular, triangular, etc...). However, we show here that this is not the best choice when addressing long-ranges, because this pulse shape leads to a significant spectral broadening of the BGS along the fibre.

An observed broadening of the BGS was suspected to be caused by self phase modulation (SPM) in an early work by Lecoeuche $e t a l^{6}$. SPM leads to small phase chirps during intensity transitions in the pump pulse (leading and trailing edges) that eventually become important in long fibres. The frequency broadening associated with this phase modulation leads to a reduced peak gain and uncertainties in the determination of the Brillouin shift $v_{\mathrm{B}}$, but leaves the temporal distribution of the pump pulse unchanged and hence the spatial resolution is preserved. While the former ${ }^{6,7}$ works showed a correct intuition addressing qualitatively the issue, we present here a quantitative model of the detrimental impact of SPM supported by a clear experimental demonstration. Three optical pulse temporal profiles were judiciously chosen (rectangular, triangular and Gaussian) showing the same FWHM width and carrying the same energy, to evenly study and compare the SPM impact on their spectrum. Then we clearly experimentally demonstrate the spectral broadening of the BGS due to SPM in a Brillouin distributed sensor in various conditions in terms of pump pulse temporal profiles, power and width.

\footnotetext{
* stella.foalengmafang@epfl.ch; phone 4121693 7387; fax 41216934660
}

Fourth European Workshop on Optical Fibre Sensors, edited by José Luís Santos, Brian Culshaw, José Miguel López-Higuera, William N. MacPherson, Proc. of SPIE Vol. 7653, 76532U

(c) 2010 SPIE $\cdot$ CCC code: $0277-786 X / 10 / \$ 18 \cdot$ doi: $10.1117 / 12.866480$ 

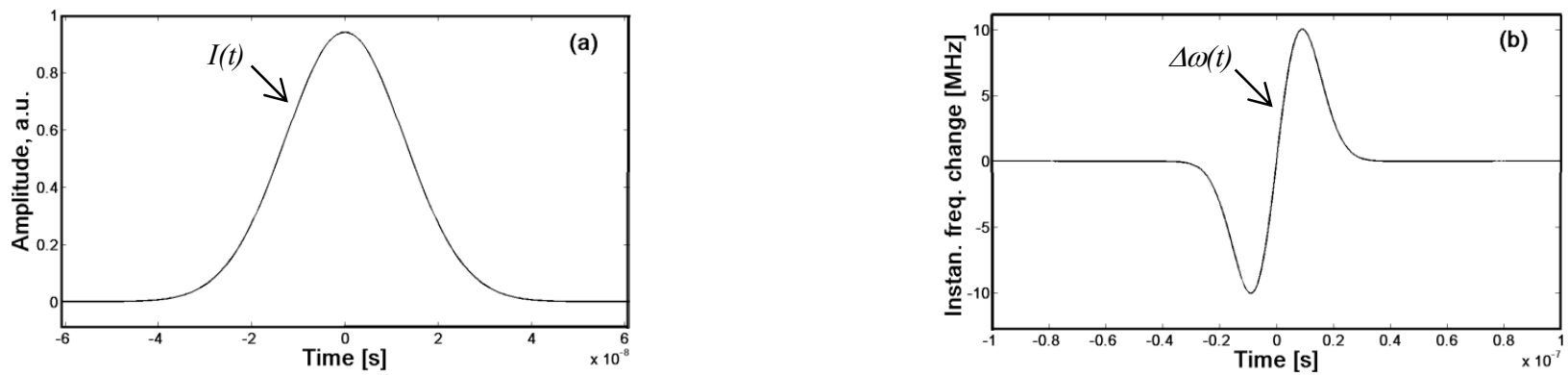

Figure 1. (a) Intensity profile of a Gaussian pulse with 30 ns FWHM width, (b) Instantaneous frequency variation due to the SPM for a peak power $P_{o}=8 \mathrm{~mW}$ and an effective length $L_{e f f}=22 \mathrm{~km}$, in a standard single mode fibre.

\section{THEORY}

SPM is a nonlinear effect which is observed in an intense time-dependent fast optical signal as it travels through an optical fibre, and is a consequence of the nonlinear response of the medium polarization that results in an intensitydependent refractive index. It leads an optical pulse to modulate its own optical phase according to its intensity profile, since the pulse will create a time-dependent refractive index variation, higher for larger intensities, leading to a timedependent nonlinear phase shift $\phi_{N L}$ that translates into variations of the instantaneous frequency (frequency chirp). Let us consider the propagation of an optical pulse through a fibre characterized by a nonlinear refractive index $n(t)=n_{0}+n_{2} I(t)$. After propagation over a distance $z$, the optical pulse accumulates an additional nonlinear phase ${ }^{8}$ due to the intensity-dependent part $\Delta n(t)=n_{2} I(t)$ of the refractive index which creates a variation in its instantaneous frequency:

$$
\Delta \omega(t)=\frac{d \phi_{N L}(z, t)}{d t}=-n_{2} \frac{\omega}{c_{0}} z \frac{d I(t)}{d t}
$$

Eq. 1 clearly shows that SPM will have more impact on fast signals constantly showing temporal transitions, with no interval showing a constant intensity. For our study we have chosen three different temporal profiles showing the same width (to secure equal spatial resolutions) and the same energy (to secure equal gain) to make even comparisons: rectangular offering constant intensity intervals only, triangular and Gaussian for which the intensity temporal profile varies constantly. Since the intensity is constant at any time for the rectangular profile (assuming instantaneous transitions), $\Delta \omega(t)=0$ and no pulse spectral broadening should therefore be observed. The situation will be drastically different in the two other cases showing uniquely time transitions. Since it can be entirely described analytically, let consider a pulse with a Gaussian envelope (Fig. 1a), $E(0, t)=A \exp \left(-t^{2} / \tau^{2}\right)$, so $I(t)=\left|A \exp \left(-t^{2} / \tau^{2}\right)\right|^{2}=I_{0} \exp \left(-2 t^{2} / \tau^{2}\right)$, with a $1 / e$ width $\tau$ in amplitude corresponding to a power FWHM width $T=\tau \sqrt{2 \ln 2}$. The instantaneous frequency variation of the Gaussian pulse due to SPM can be written as:

$$
\Delta \omega(t)=4 n_{2} \frac{\omega}{c_{0}} z I_{0} \frac{t^{2}}{\tau^{2}} \exp \left(-2 t^{2} / \tau^{2}\right)=4 \gamma z P \frac{t}{\tau^{2}} \exp \left(-2 t^{2} / \tau^{2}\right)
$$

where $\omega$ is the central angular frequency of the optical pulse, $I_{o}$ and $P$ are, respectively, the peak intensity and peak power of the optical pulse, $A_{\text {eff }}$ is the effective area of the fibre mode and $\gamma=n_{2} \omega / c_{0} A_{\text {eff }}$ is the fibre nonlinear coefficient. In a long fibre segment the position $z$ must be replaced by the effective nonlinear length $L_{\text {eff }}=[1-\exp (-\alpha L)] / \alpha$ where $L$ is the physical length and $\alpha$ in the linear loss of the fibre. As shown in Fig. 1b, the linear frequency variation (chirp) imposed on the central part of the Gaussian pulse is of the order of $\pm 10 \mathrm{MHz}$ for $P_{o}=8 \mathrm{~mW}$, $T=30 \mathrm{~ns}$ and $L_{e f f}=22 \mathrm{~km}$. The peak excursion of the instantaneous frequency is $\Delta \omega=2 \gamma P L_{e f f} / \sqrt{e} \tau=1.43 \gamma P L_{e f f} / T$ 

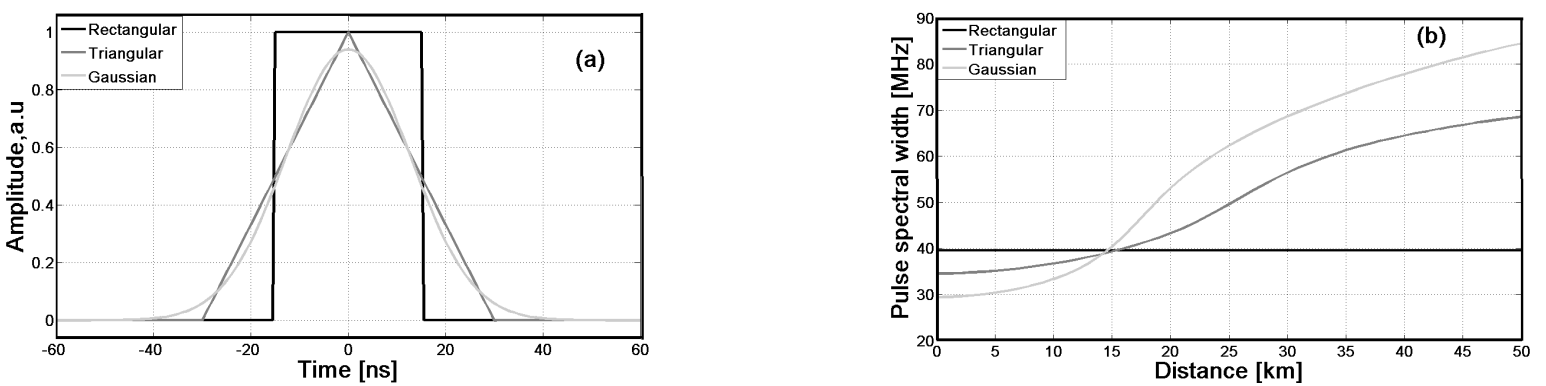

Figure 2. (a) Scaled intensity profiles used for the study, showing identical $30 \mathrm{~ns}$ FWHM width and energy, (b) Corresponding FWHM spectral width as a function of the propagation distance $\left(P=100 \mathrm{~mW}, \gamma=1.4 \mathrm{~W}^{-1} \mathrm{~km}^{-1}\right)$, illustrating the profiledependent SPM broadening.

offset by $\tau_{\max }=T / \sqrt{8 \ln 2}$ from the pulse centre and, as scale factor, gives a good estimate of the real spectral broadening. The real frequency spectrum of the Gaussian pulse after SPM is given by the Fourier transform of the output pulse amplitude given by the following expression:

$$
\underbrace{A \exp \left(-t^{2} / \tau^{2}\right)}_{\text {Gaussian amplitude term }} \underbrace{\exp \left[i \gamma L_{\text {eff }} P \exp \left(-2 t^{2} / \tau^{2}\right)\right]}_{\text {SPM term }}
$$

where the time origin is always placed at the pulse centre during the propagation.

Fig. 2a represents the temporal distribution of the three pulse intensity profiles, scaled to present the same $30 \mathrm{~ns}$ FWHM width and to carry the same energy. In the frequency domain the calculation of the pulse spectral width (FWHM) as a function of the distance shows that the output spectrum for a rectangular pulse remains unchanged (Fig. $2 \mathrm{~b}$ ), as predicted. As a result of SPM, the pulse shows after $50 \mathrm{~km}$, on top of the original $35 \mathrm{MHz}$ width, an excess spectral broadening of some $35 \mathrm{MHz}$ for the triangular case and of $55 \mathrm{MHz}$ for the Gaussian case, as illustrated in Fig. $2 b$.

\section{EXPERIMENTAL DEMONSTRATION}

\subsection{Experimental implementation}

The experimental configuration, shown in Fig. 3 , is based on a minor adaptation of the high performance pump and probe set-up described in 9 . A $1552 \mathrm{~nm}$ DFB laser is used as the light source. The output of the laser diode is split into two distinct channels to allow both the pump and signal waves to be derived from the same optical source ${ }^{10,11}$. The signal is generated in the upper channel, where an integrated electro-optic intensity modulator, driven by a microwave signal, creates sidebands in a suppressed carrier configuration, which are launched into one end of the sensing fibre. The lower channel is connected to the opposite end of the sensing fibre in which the laser light, periodically

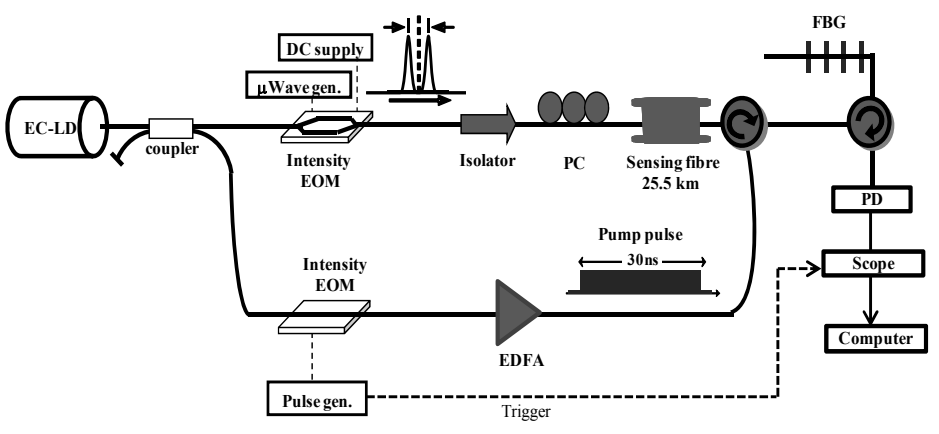

Figure 3. Diagram of the setup: EC-LD: external cavity laser diode; EDFA: erbium-doped fibre amplifier; EOM: electro-optic modulator; FBG: fibre Bragg grating; PD: photodiode. modulated by an intensity modulator to produce pump pulses, is launched. An arbitrary waveform generator is 

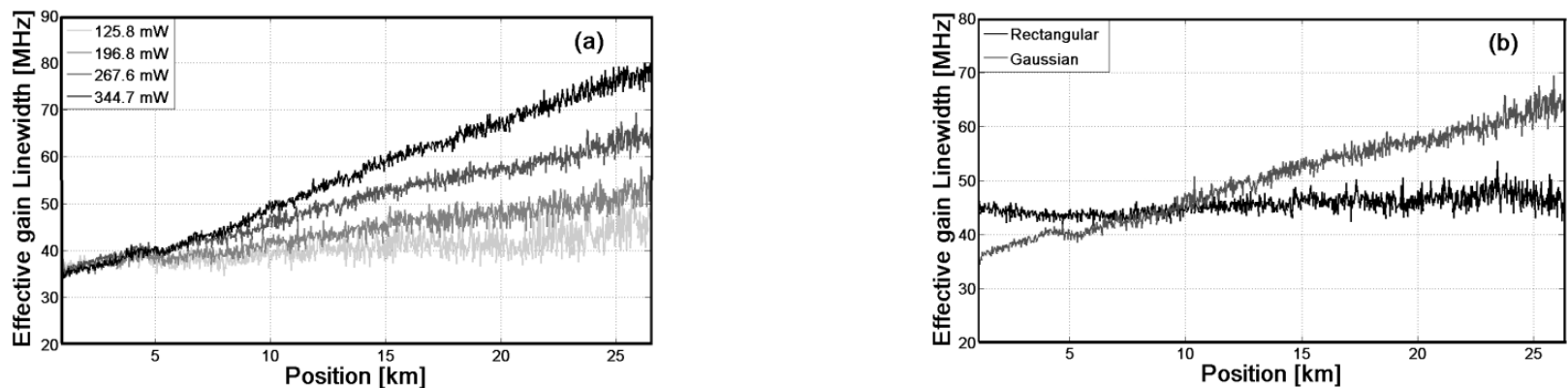

Figure 4. Experimental demonstration of the gain spectrum broadening due to SPM along $25 \mathrm{~km}$ SMF fibre (a) for different peak powers of a 30 ns FWHM Gaussian pulse (b) comparing 30 ns FWHM Gaussian and rectangular pulses of identical energy.
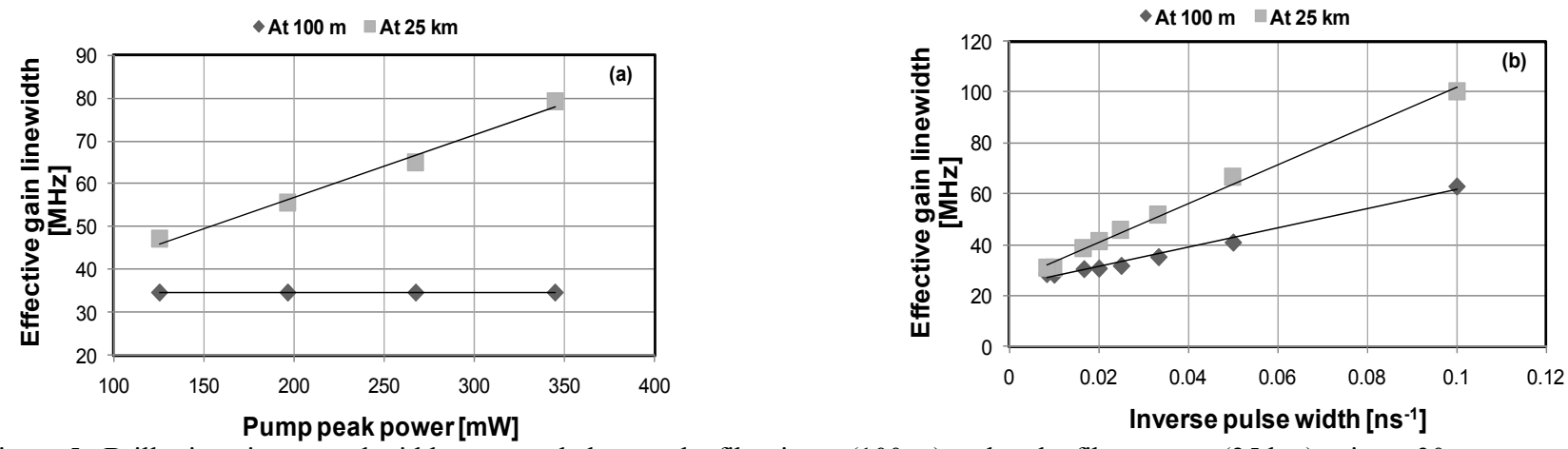

Figure 5. Brillouin gain spectral width measured close to the fibre input $(100 \mathrm{~m})$ and at the fibre output $(25 \mathrm{~km})$ using a $30 \mathrm{~ns}$ Gaussian pulse (a) Linear dependence on the pump peak power, showing that the effect of SPM is observed only at the distant end,

computer-controlled to deliver different pulse temporal profiles which are then applied to the intensity modulator, resulting in $30 \mathrm{~ns}$ FWHM optical pulse duration corresponding to $3 \mathrm{~m}$ spatial resolution. This duration was carefully chosen for a better demonstration as a trade-off between temporal transitions fast enough to show the SPM effect and long enough to keep the original spectrum sufficiently narrow to avoid an excessive broadening of the effective Brillouin gain spectrum. The output light is controlled by a polarization controller to best align pump and signal polarizations to maximize the SBS interaction, and then boosted by a high power EDFA. The Brillouin gain spectrum is determined by scanning the microwave generator frequency around the Brillouin shift $v_{B}$. The net Brillouin gain is then measured by detecting the optical signal using a DC-125 MHz photoreceiver, which is then acquired by a digital oscilloscope synchronously triggered by the arbitrary waveform generator.

\subsection{Experimental results}

The experimental study of the SPM impact on Brillouin sensors has been carried out through a $25 \mathrm{~km}$ SMF fibre using the above described BOTDA system. The distributed nature of the measurements makes possible to retrieve the effective Brillouin gain spectrum at any position along the fibre, and thus to observe the gradual impact of SPM. Fig. 4a shows the effective gain linewidth along the fibre using a $30 \mathrm{~ns}$ FWHM Gaussian pulse for increasing pump peak power from $125.8 \mathrm{~mW}$ to $344.7 \mathrm{~mW}$, showing the evidence of the gain spectral broadening due to SPM along the fibre. Fig. $4 \mathrm{~b}$ represents the effective gain linewidth of the fibre using $30 \mathrm{~ns}$ FWHM rectangular and Gaussian pulse of identical energy (267.6 $\mathrm{mW}$ Gaussian peak power). It can be observed that the gain spectral width is doubled after $25 \mathrm{~km}$ for a Gaussian pulse, while it remains unchanged for the rectangular pulse. The benefit of a better timebandwidth product using the Gaussian pulse turns out to be entirely cancelled by SPM after less than $10 \mathrm{~km}$. Fig. 5a 
represents comparative measurements of the gain spectral width measured for a Gaussian pulse at the fibre input (black curve) and output (gray curve), respectively, for different peak powers, demonstrating the clear signature of SPM by the absence of effect at short distance and through the linear dependence on power at long distances. As shown in Fig. $5 \mathrm{~b}$ the effective Brillouin gain linewidth varies in inverse proportion to the optical pulse duration $T$ at both the fibre input and output, but with a different slope. While a natural excess broadening is present as a result of the pump spectral spreading for shorter pulses, illustrated by slope of the $1 / T$ dependence at the fibre input (black curve), SPM modulation induces an extra broadening with the same $1 / T$ dependence that results in a larger slope (gray curve) that turns gradually steeper for longer effective length.

\section{CONCLUSION}

We have demonstrated that SPM-induced spectral broadening can have a significant effect on the effective gain linewidth measured by Brillouin sensors. Modeling and experiments have undoubtedly demonstrated that the effective gain linewidth can easily experience a two-fold increase in standard conditions when the pulse intensity profile constantly shows temporal variations like in the triangular and Gaussian cases. At power and spatial resolutions used in standard configurations, spectral broadening can be observed typically from a $5 \mathrm{~km}$ distance. Practically the problem can be circumvented by using a very clean rectangular pulse with very sharp rising and falling edges. The benefit of a narrower spectrum for an identical pulse width brought by the Gaussian pulse is rapidly cancelled after about $10 \mathrm{~km}$.

\section{REFERENCES}

1. T. Kurashima, T. Horuguchi and M. Tateda, Opt. Lett. 15 (18), 1038-1040 (1990).

2. X. Bao, D.J. Webb, and D.A. Jackson, Opt. Lett. 18 (18), 1561-1563, (1993).

3. M. Nikles, L. Thévenaz, and P. A. Robert, Opt. Lett. 21 (10), (1996).

4. L. Thévenaz and S. M. Foaleng, OFS $19^{\text {th }}$, Proc. SPIE 70043N-70044, Perth, WA, Australia, (2008).

5. S. M. Foaleng, J-C Beugnot, L. Thévenaz, OFS $20^{\text {th }}$, Proc. SPIE 7503, 75032C-4, Edinburgh, UK, Scotland, (2009).

6. V. Lecoeuche, D.J. Webb, C.N. Pannell, D.A. Jackson, Opt Com, 168, 95-102 (1999).

7. J.W. Czarske, I. Zawischa, A. Tünnermann, Proc. OFS OThC33, 536-539, Washington DC, USA, (1997).

8. R. W. Boyd, [Nonlinear Optics], fourth edition, Chap.7 (2008).

9. S. Diaz, S. M. Foaleng, M. Lopez-Amo, and L. Thévenaz, IEEE Sensors journal 8, 1268-1272 (2008).

10. M. Niklès, L. Thévenaz and P. Robert, JLT 15 (10), 1842-1851, (1997). 